From representing to modelling knowledge: Proposing a two-step training for excellence in concept mapping

\author{
Joana G. Aguiar \\ Paulo R. M. Correia
}

Universidade de São Paulo, SP, Brazil

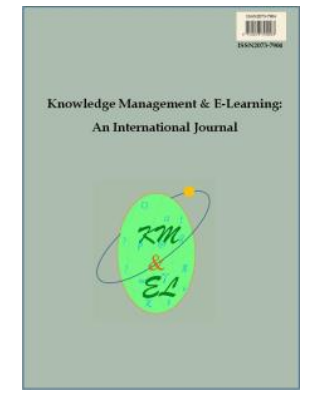

Knowledge Management \& E-Learning: An International Journal (KM\&EL) ISSN 2073-7904

Recommended citation:

Aguiar, J. G., \& Correia, P. R. M. (2017). From representing to modelling knowledge: Proposing a two-step training for excellence in concept mapping. Knowledge Management \& E-Learning, 9(3), 366-379. 


\title{
From representing to modelling knowledge: Proposing a two-step training for excellence in concept mapping
}

\section{Joana G. Aguiar}

Programa de Pós-graduação Interunidades em Ensino de Ciências

Universidade de São Paulo, SP, Brazil

E-mail: joanaguilares@usp.br

\author{
Paulo R. M. Correia* \\ Escola de Artes, Ciências e Humanidades \\ Universidade de São Paulo, SP, Brazil \\ E-mail: prmc@usp.br
}

*Corresponding author

\begin{abstract}
Training users in the concept mapping technique is critical for ensuring a high-quality concept map in terms of graphical structure and content accuracy. However, assessing excellence in concept mapping through structural and content features is a complex task. This paper proposes a two-step sequential training in concept mapping. The first step requires the fulfilment of low-order cognitive objectives (remember, understand and apply) to facilitate novices' development into good Cmappers by honing their knowledge representation skills. The second step requires the fulfilment of high-order cognitive objectives (analyse, evaluate and create) to grow good Cmappers into excellent ones through the development of knowledge modelling skills. Based on Bloom's revised taxonomy and cognitive load theory, this paper presents theoretical accounts to (1) identify the criteria distinguishing good and excellent concept maps, (2) inform instructional tasks for concept map elaboration and (3) propose a prototype for training users on concept mapping combining online and face-to-face activities. The proposed training application and the institutional certification are the next steps for the mature use of concept maps for educational as well as business purposes.
\end{abstract}

Keywords: Concept mapping; Knowledge representation; Knowledge modelling; Proficiency; Certification

Biographical notes: Joana Aguiar has been part of the concept mapping research group at University of São Paulo since 2010. She gained an MSc in Science Teaching and is currently finishing her doctoral thesis focusing on 'map shock' - excessive cognitive load due to the shape and/or content caused by concept maps prepared by Chemistry teachers. Joana was part of the organising and scientific committees of the Sixth International Conference on Concept Mapping (CMC2014) organised by USP and IHMC.

Paulo Correia is Professor within the School of Arts, Science and Humanities at the University of São Paulo. He has been involved in research on concept mapping applied to teaching and learning since 2006. His current research aims to understand how to optimise the use of concept mapping in considering human cognitive architecture. Paulo was the chairman of the Sixth International Conference on Concept Mapping (CMC2014) organised by USP and IHMC. 


\section{Introduction}

Concept maps are graphical tools for organising and representing knowledge through propositions - that is, the relationships between pairs of concepts by a linking line and phrase. Concepts are usually hierarchically organised, and the entire propositional network answers a specific focus question (Cañas \& Novak, 2006). According to Cañas, Novak, and Reiska (2015), several main factors distinguish between good and excellent concept maps (Fig. 1). Good concept maps must fulfil some predefined criteria related to both graphical structure and content accuracy, such as answering a focus question, organising concepts in a hierarchical way and constructing correct and relevant propositions. However, these criteria do not ensure an excellent concept map, which is not only concise but also capable of capturing the complexity of the involved content. Concept map quality is related to Cmappers' (Cmapper is the person who creates/elaborates the concept map) proficiency in the technique and their expertise in the content. However, assessing this quality is a complex issue. Cañas, Novak, and Reiska (2015) highlight the difficulty of recognising excellent concept maps:

An excellent concept map is like a good poem, we know when we have read one, but we cannot quantify the reason. Professional Cmappers can recognise them, but it is hard to teach how to construct them. (Cañas, Novak, \& Reiska, 2015, p. 17)

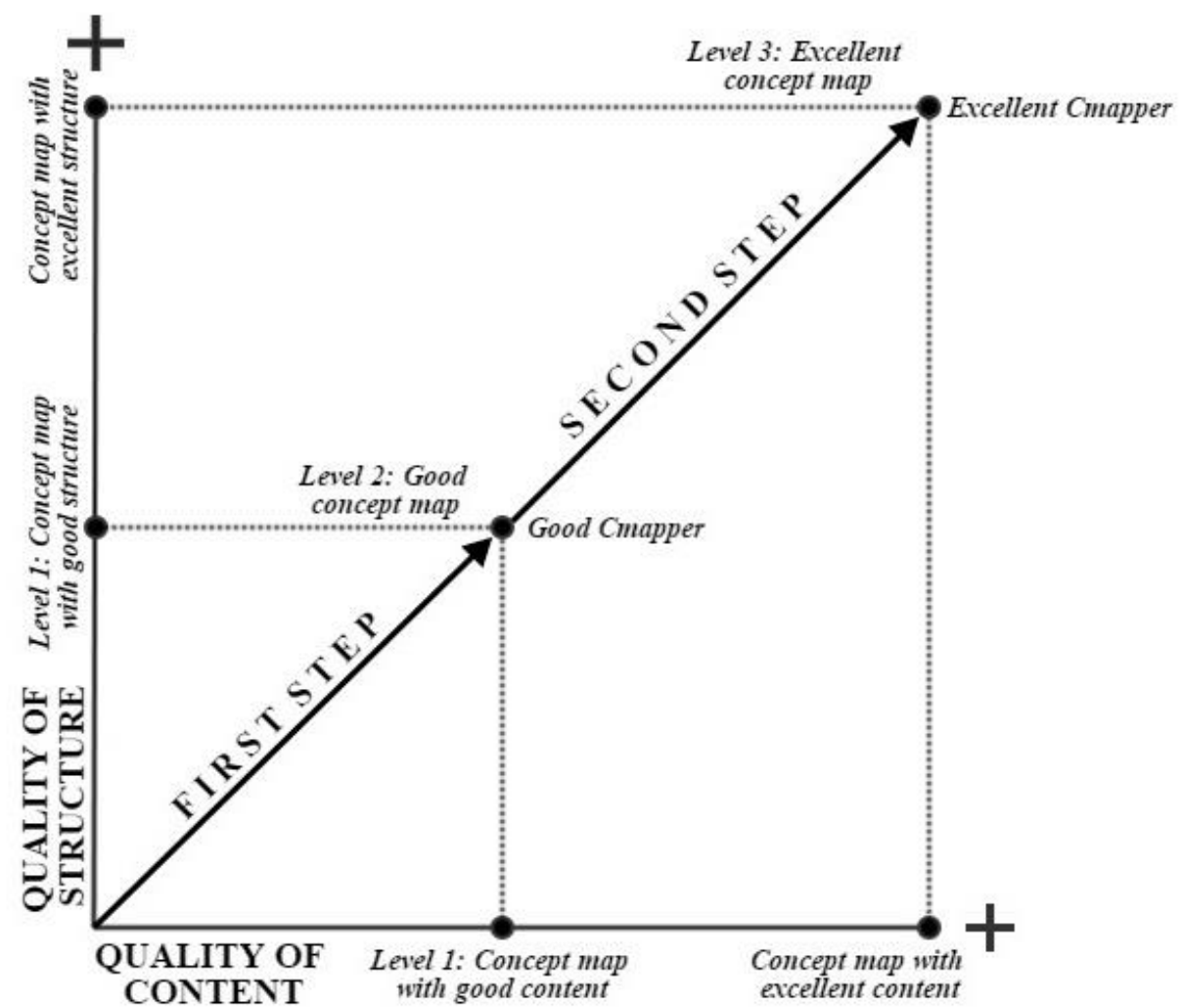

Fig. 1. Graphical structure and content accuracy are both criteria that define concept map quality. Good and excellent Cmappers can be distinguished by the extent to which they can handle the criteria to produce good or excellent concept maps. Adapted from Cañas, Novak, \& Reiska (2015). 
The difficulty in assessing concept map quality permeates important questions, which remain without answers, such as how someone can become an excellent Cmapper and which competencies must be developed throughout this process, what the main features of excellent concept maps are, and how large they should be (Cañas, Reiska, \& Novak, 2016). Our research group has carried out some studies to develop and apply training activities to improve Cmappers' proficiency (Aguiar, Cicuto, \& Correia, 2014). Cognitive load theory offers a theoretical background that informs the best instructional strategies in this training process (Correia \& Aguiar, 2014). We succeeded in implementing instructions and exercises in real classrooms to train novices to become good Cmappers through improvements in their knowledge representation. Thus far, the results are promising, and a large-scale implementation is conceivable considering a computer-mediated intervention.

This paper proposes a two-step sequential training to form excellent Cmappers (Fig. 1). The first step focuses on online and computer-mediated activities to train novices in knowledge organisation and representation (Table 1). The second step considers face-to-face activities for learning how to model knowledge through concept maps, leading Cmappers to develop excellence in the technique (Table 1). We address three main theoretical accounts:

- Distinction between good and excellent concept maps through their benchmarks

- Description of instructional tasks needed to accomplish the training phases

- Proposal of a certification-oriented course prototype to achieve excellence.

Table 1

Main features that distinguish the two-step sequential training proposed in this paper

\begin{tabular}{lll}
\hline & First Step & Second Step \\
\hline Format & Online and computer-mediated & Face-to-face \\
Focus & Knowledge representation & Knowledge modelling \\
Level of expertise & Novice to good Cmapper & Good to excellent Cmapper \\
\hline
\end{tabular}

\section{Distinction between good and excellent concept maps: Benchmarks for quality and proficiency assessment}

Concept maps are constituted by two main features: graphical structure and semantic content. The structure is related to the visual layout aspects (Buhmann \& Kingsbury, 2015), such as location and amount of concepts and propositions, hierarchical arrangement of concepts, legibility and propositional flow, the quantity of arrows indicating the reading pathway, and the presence of cross-links as well as navigation visual cues. On the other hand, the semantic content accuracy is related to the validity and pertinence of concepts, linking phrases and propositions, the presence and quality of given examples as well as the extent to which the hierarchy, focus question and crosslinks are accurate and suitable considering the topic. Both features raise solid criteria for assessing concept maps' quality, which can be summarised into four key elements of the mapping technique (Novak \& Cañas, 2008; De Aguiar \& Correia, 2013):

- The propositions must have high semantic clarity and also communicate the conceptual relationship accurately 
- The focus question bounds the topic to be mapped and also controls the size of the map

- The conceptual organisation should present a hierarchy, meaning the most inclusive concepts begin the concept map and are progressively detailed

- Continuous revisions should be used to modify the represented knowledge according to the changes in the Cmappers' understanding about the topic

Table 2 presents a combination of these four key elements regarding their contribution to enhance graphical structure or semantic content accuracy. It also provides the benchmarks needed to distinguish good from excellent concept maps. Good concept maps meet the criteria established on the diagonal (in black) while excellent ones meet all the criteria. To become a good Cmapper, individuals must incorporate these elements into the concept map as soon as they learn about them. Hilbert and Renkl (2008) showed that learning concept mapping successfully involves the engagement in a circular process with three steps - planning, constructing, controlling - and, as necessary, revising and planning again. In sum, continuous revision is an important strategy for ensuring the best representation of knowledge.

Fig. 2 illustrates an example of how to construct a good concept map about the topic "research paper". First, the most important concepts (events or objects) are listed considering the topic (Fig. 2a). Second, the concepts are hierarchically organised (Fig. 2b). The Cmapper can now choose the branch that he/she desires to develop in the concept map. In our example, we chose to represent papers' structure, setting aside the concepts related to the submission process (in grey). Third, Cmappers have to construct the propositions, paying special attention to the relationship between the concepts through correct and complete linking phrases (Fig. 2c). Finally, the entire network should be read, followed by the statement of a proper focus question (Fig. 2c). This process ensures good knowledge representation, from the sum of the parts (propositions made up of concepts hierarchically organised) to the whole (a good concept map that answers a specific focus question). Good Cmappers can be assessed on how well they are capable of producing good concept maps by handling these key elements.

Excellent concept maps fulfil not only the criteria given in the diagonal (Table 2) but also the others boxes. To become an excellent Cmapper, individuals have to understand and handle all the key elements considering their multiple combinations. For instance:

- $\quad$ Focus question modifications guide the choice of new concepts and propositions (combination between focus question/content and proposition/structure)

- The hierarchy may be reconsidered when a different focus question is stated (combination between focus question/content and hierarchy/structure)

- Cross-links are identified more easily when the hierarchy highlights subdomains of knowledge (combination between hierarchy/content and proposition/structure)

Continuous revision is an important strategy for ensuring the modelling of knowledge. Fig. 2d illustrates an example of how to construct an excellent concept map on the "research paper" topic. It is critical to ensure that the knowledge is already organised and represented (as shown in Fig. 2c) before starting modelling. The establishment of a more specific focus question opened the possibility to: 
- $\quad$ Look for cross-links (e.g., 'discussion $\rightarrow$ should offer comparative studies found in a $\rightarrow$ literature review')

- Check the pertinence of concepts (e.g. 'final consideration' instead of 'conclusion')

- Reorganise the conceptual hierarchy

- Check the layout and reading flow

- Add examples (boxes with dashed lines)

- Improve linking phrases to answer and explain the focus question

- Include graphical cues (e.g., numbered concepts to offer a reading sequence)

\section{TOPIC: RESEARCH PAPERS}

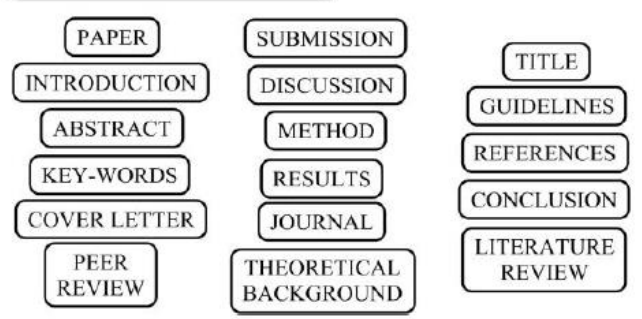

Fig. 2a

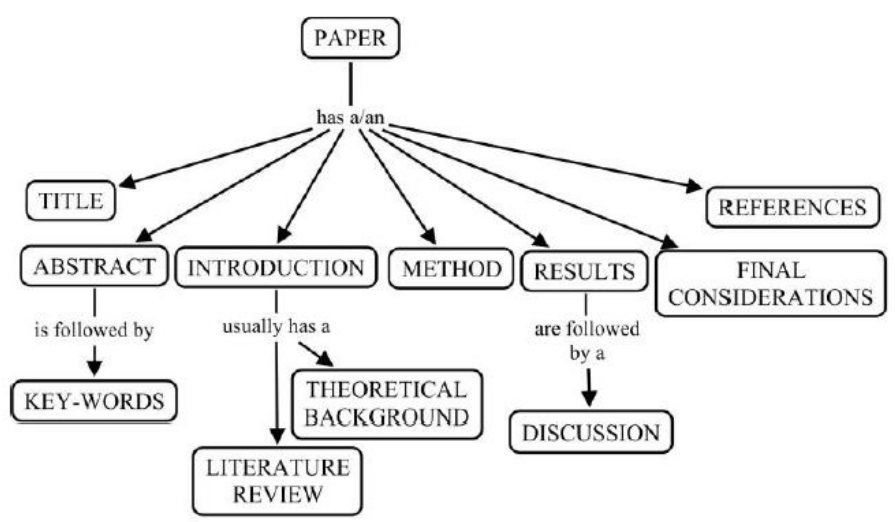

Focus Question: What is the general structure of a research paper?

Fig. 2c

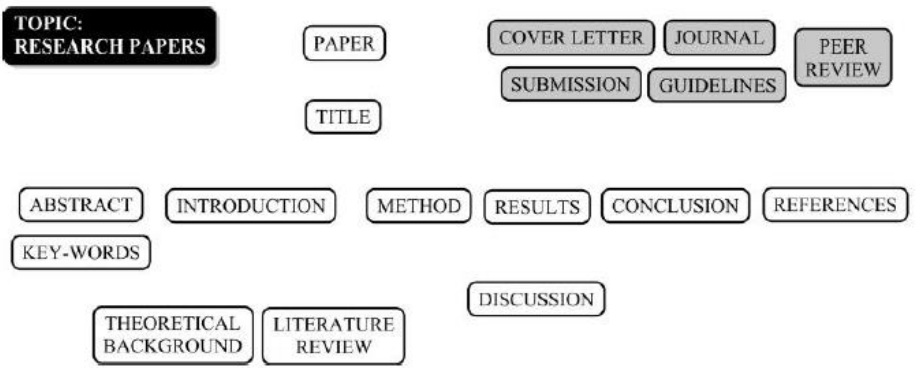

Fig. 2b

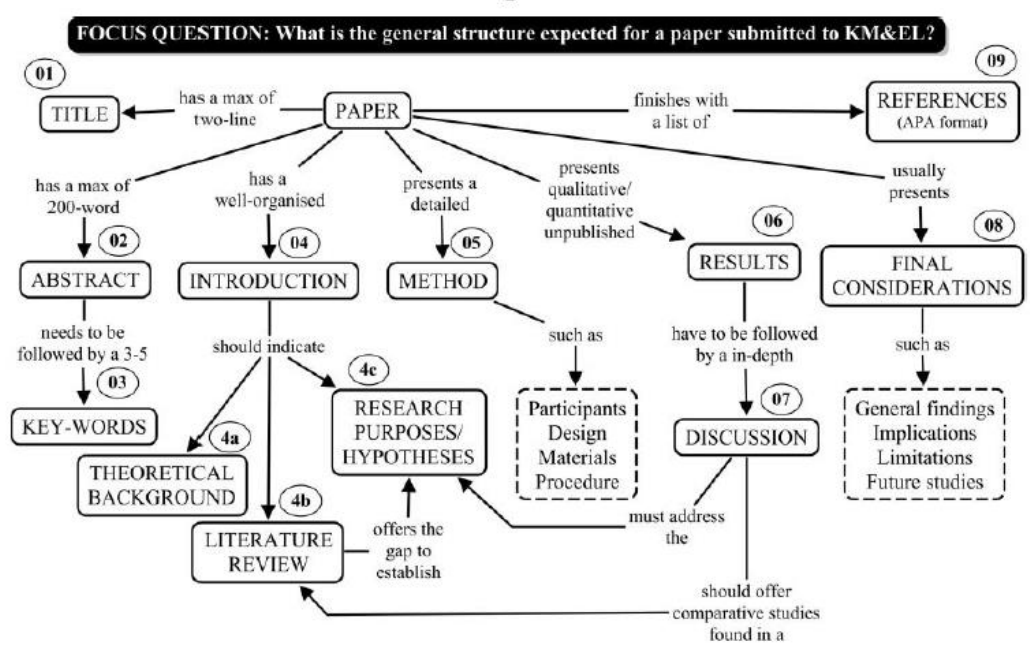

Fig. 2d

Fig. 2. How to construct a good concept map about the topic "research paper" by (a) listing concepts, (b) organising the concepts hierarchically, and (c) establishing clear and correct propositions as well as a proper focus question. Modelling the represented knowledge would lead to (d) an excellent map. 


\section{Table 2}

Four concept map benchmarks described as criteria for content accuracy and/or graphical structure qualities. Good concept maps fulfil the requirements described along the diagonal (in black). Excellent ones fulfil all the requirements.

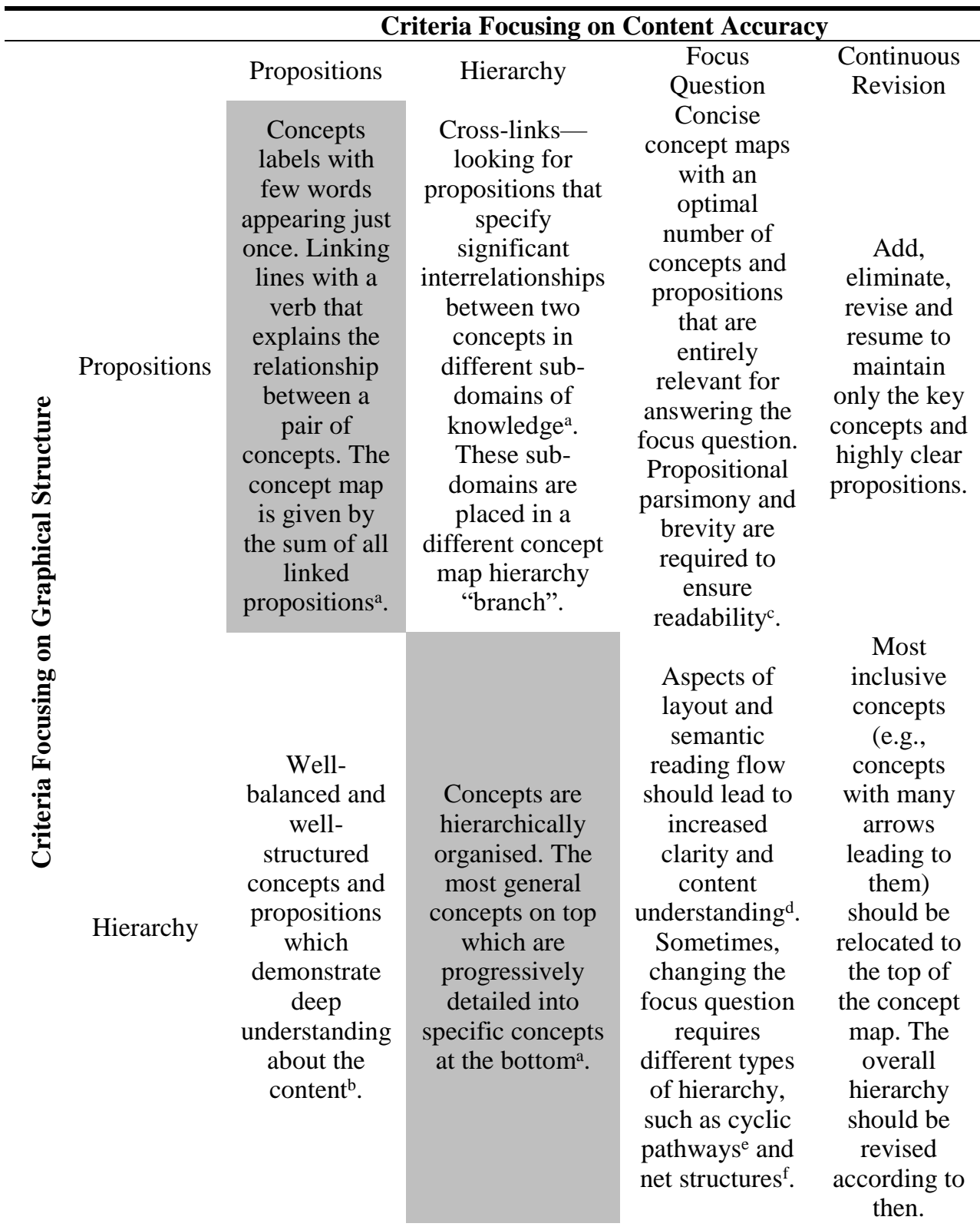




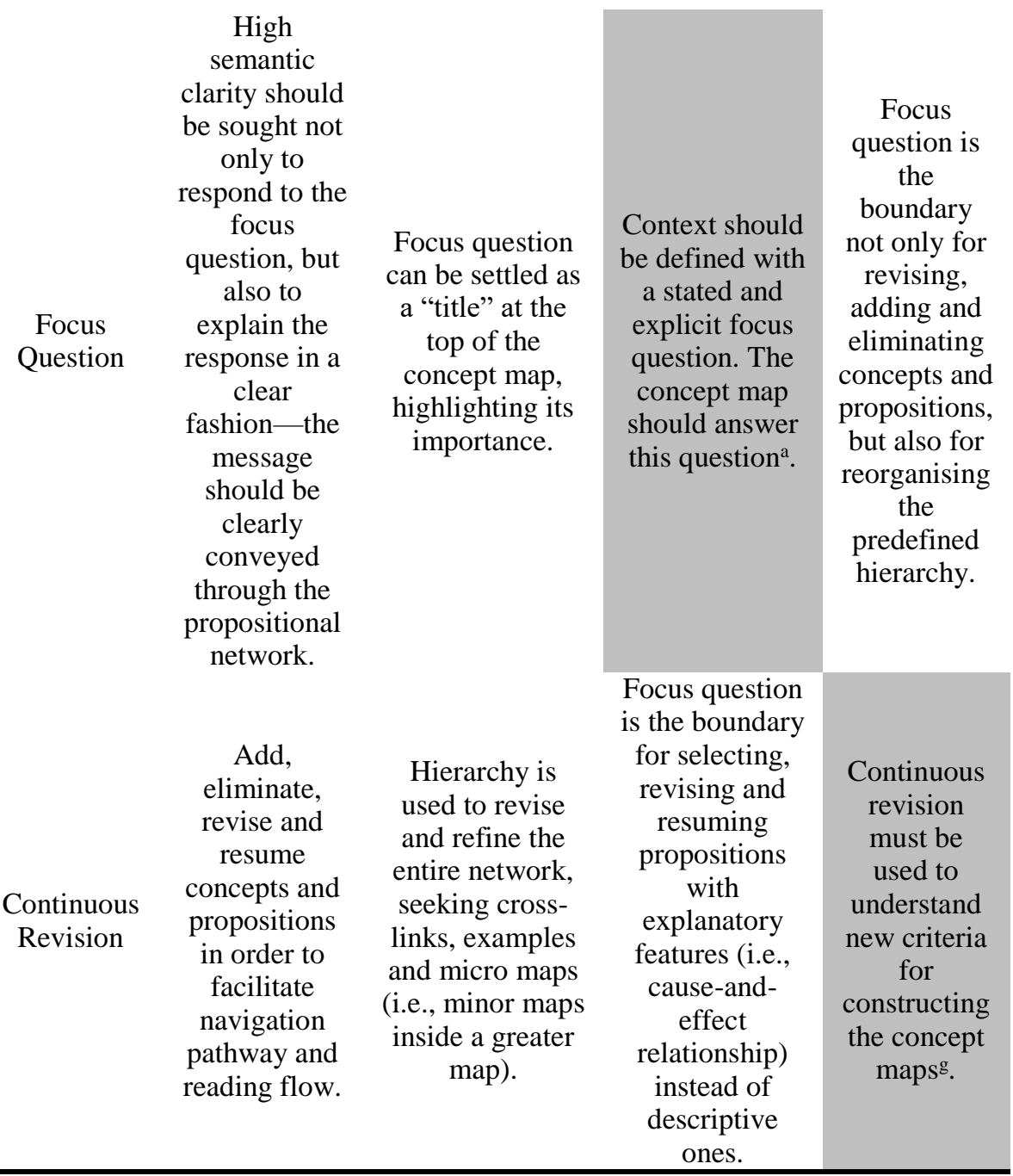

Note. ${ }^{\mathrm{a}}$ Novak \& Cañas, 2008. ${ }^{\mathrm{b}}$ Carvajal, Cañas, Carballeda, \& Hurtado, 2006. ${ }^{\mathrm{c}}$ Derbentseva \& Kwantes, 2014. ${ }^{\mathrm{d}}$ Buhmann \& Kingsbury, 2015. ${ }^{\mathrm{e}}$ Safayeni, Derbentseva, \& Cañas, 2005. ${ }^{\mathrm{f}}$ Kinchin, Hay, \& Adams, 2000. ${ }^{\mathrm{g}}$ De Aguiar \& Correia, 2013.

This process allows the refinement of the whole (a concept map more concise and indicative of the content complexity) through the relationships among the parts (combination of the key elements). An excellent Cmapper can be assessed on how well he/she is capable of producing excellent concept maps by handling multiple combinations of the key elements to ensure knowledge modelling, resulting in a high quality of structure and content.

\section{Training tasks informed by Bloom's revised taxonomy and cognitive load theory}

For the past two decades, concept mapping has been used as a tool to aid knowledge management. This process requires knowledge organisation, representation, refinement 
and sharing (Newbern \& Dansereau, 1995; Cañas, Leake, \& Wilson, 1999; Fischer \& Mandl, 2001; Coffey, Hoffmann, Cañas, \& Ford, 2002; Tergan, 2005; Hoffman, Ziebell, Fiore \& Becerra-Fernandez, 2008; Novak, 2010; Correia, 2012). In this paper, we propose a training approach that splits the skills for knowledge management through concept maps into two consecutive stages:

- $\quad$ Organise and represent knowledge

- $\quad$ Refine knowledge (also known as modelling)

Each stage requires distinct cognitive processes as well as the development of specific abilities. Bloom's revised taxonomy (Anderson \& Krathwohl, 2001) helps classify the objectives to be fulfilled in each training step (Fig. 3). For instance, one of the first requirements for becoming a good Cmapper is constructing propositions by putting together two concepts and a linking phrase with a well-conjugated verb. To achieve this goal, the Cmapper must (1) remember that concept labels have one or a few words, (2) understand the meaning of the proposition and (3) apply the procedure to unite two concepts and a verb that explains their relationship. In this case, the Cmapper has to grasp not only the propositional structure, but also the content to be mapped (see the relationship between proposition/structure and proposition/content in Table 2).

A good concept map and, consequently, a good Cmapper can be evaluated by checking the extent to which he/she was able to meet the criteria for propositions:

- Correctness of the propositional structure (i.e., two concepts, an arrow that guides the reading flow and the presence of a verb in the linking phrase)

- Correctness of the linking phrase (i.e., a valid and accurate conceptual relationship considering the expert view)

One of the first requirements for becoming an excellent Cmapper is to construct a more concise concept map with an optimal number of concepts and propositions that are entirely relevant for answering the focus question. Propositional parsimony and brevity are required to ensure readability (Derbentseva \& Kwantes, 2014). To achieve this goal, the Cmapper must (1) evaluate the pertinence and validity of concepts and propositions regarding the focus question, (2) analyse the extent to which the focus question is explained every time that the propositions are changed and (3) create a new propositional network which is more concise and coherent considering all the changes. In this case, the Cmapper has to consider the focus question to enhance the content accuracy by manipulating the propositional structure (see the relationship between proposition/structure and focus question/content in Table 2). An excellent concept map and, consequently, an excellent Cmapper can only be evaluated considering the overall quality of the produced map. It is not possible to make specific judgements about how he/she manipulated the criteria (see all the white boxes in Table 2).

Becoming a good Cmapper involves the achievement of objectives with a low level of cognitive process, such as remember facts, understand concepts and apply rules (Fig. 3). On the other hand, excellence in concept mapping requires the achievement of objectives with a high level of cognitive process, such as evaluate according to certain criteria, analyse parts to improve the whole and create a new whole from the enhancement of the parts (Fig. 3).

Krathwohl (2002) and Mayer (2002) argue that instructional tasks which demand low-order thinking skills would hierarchically precede those of high order, ensuring a gradual increase in the subject competence and avoiding overload his/her cognitive 
system. To sum up, organising and representing knowledge through concept mapping would precede knowledge modelling, and both tasks must be planned to avoid cognitive overload.

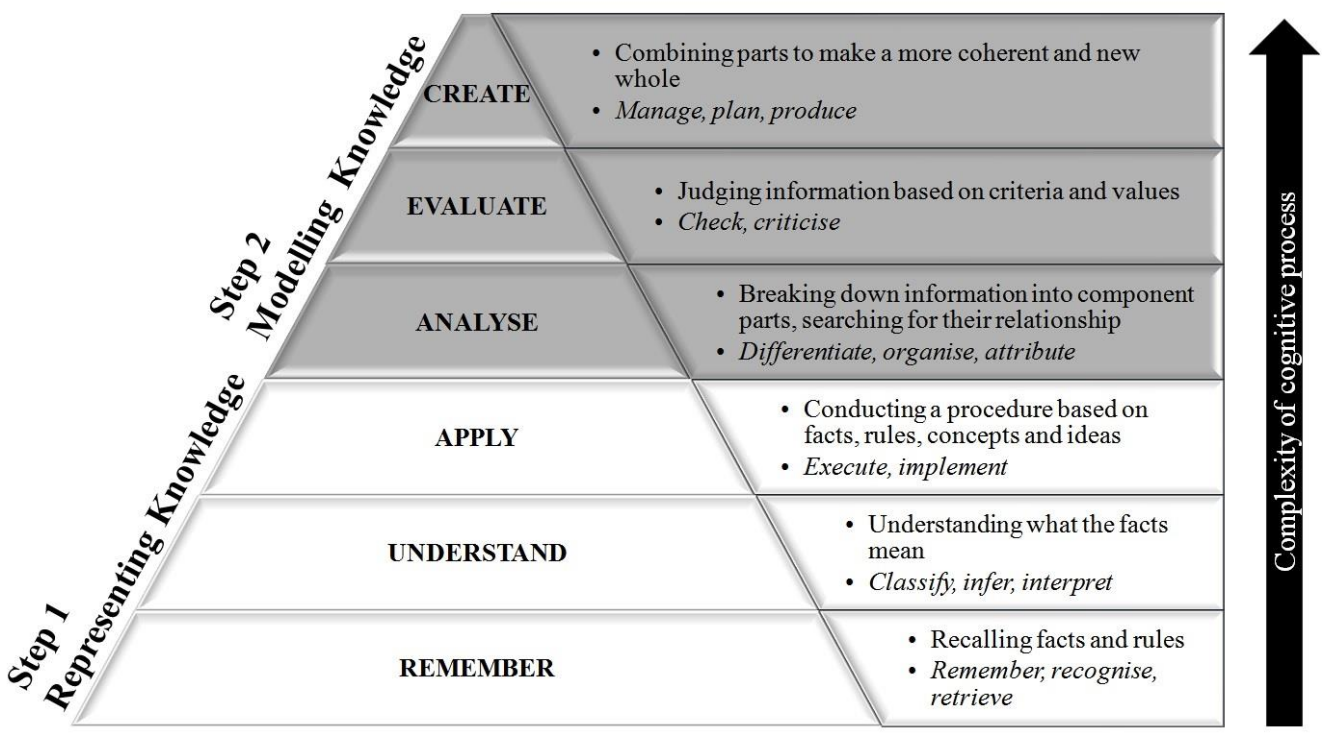

Fig. 3. Bloom's revised taxonomy considering the cognitive process dimension, which is hierarchically organised from the lowest level of complexity (remember) to the highest level (create).

According to cognitive load theory (Sweller, Ayres, \& Kalyuga, 2011), instructional tasks can be a source of two types of cognitive load:

- Intrinsic load (I) related to the content complexity - that is, the more elementary the interactivity, the more complex the content.

- $\quad$ Extraneous load $(\boldsymbol{E})$ related to instructional methods and materials used during learning tasks — that is, the more ill-structured the material, the more detrimental the learning process.

Intrinsic and extraneous loads are additives. If they exceeded the limits of working memory, then learning would be hindered once there are no resources left to foster generative processes - namely, schema construction and manipulation (van Merriënboer \& Ayres, 2005; Sweller, Ayres, \& Kalyuga, 2011; van Mierlo, Jarodzka, Kirschner, \& Kirschner, 2012).

Fig. 4 indicates how both loads change as we train subjects to use concept mapping to knowledge management. Untrained users are more susceptible to cognitive overload during concept map elaboration tasks because they need to organise a complex new content (high I load) using an unfamiliar graphic organiser (high E load). The sum of loads will surpass the subject's working memory capacity (namely, cognitive overload), leading to a low-quality concept map (poor in structure and content). Furthermore, without training, it is impossible to use concept mapping as an assessment tool once a low-quality concept map does not represent users' mental model (Correia \& Aguiar, 2014). 


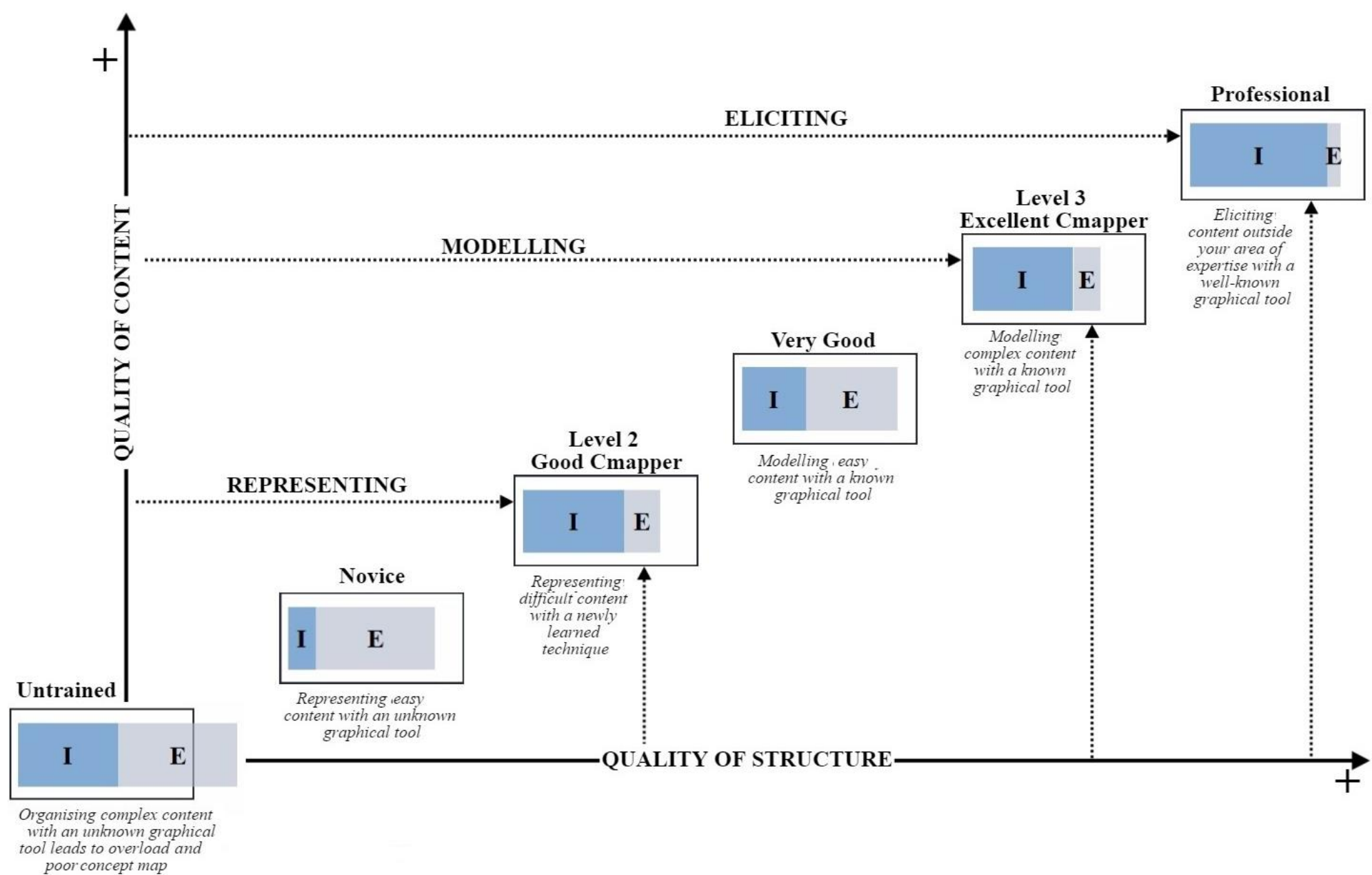

Fig. 4. Scheme to illustrate how the intrinsic cognitive load (I, related to the content complexity) and the extraneous cognitive load (E, related to task format) change during Cmap training. This paper proposes a two-step training, leading the novice to become an excellent Cmapper through the development of capabilities of representing and modelling knowledge. Professional Cmappers are capable of eliciting and preserving knowledge.

The first step of training implies learning how to represent the knowledge by managing concept map key elements as units (see the black diagonal in Table 2). This strategy decreases the I load by dropping content complexity. Consequently, more free working memory resources would be used to understand the technique, which means dealing with the imposed E load raised by an unfamiliar graphical organiser.

Once Cmappers overcome this stage, they are capable of understanding and handling the main elements of concept mapping, thereby decreasing the E load. The automation of knowledge representation through concept maps leads individuals to a good Cmapper level (Level 2). The Cmapper can map a difficult or complex content, increasing the I load. The result would be a good concept map relying on his/her mental model and able to be evaluated regarding graphical structure and content accuracy. 
Continuous revision plays a key role to avoid cognitive overload during learning stages and reconstruct knowledge over time.

Fig. 4 also shows the second step of training, which implies learning how to model knowledge by combining concept map key elements. In this process, the I load increases due to the manipulation of multiple elements during the refinement of knowledge (see the white boxes in Table 2). At the same time, the E load also increases as an effect of handling the technique during a task that is way more complex than representing knowledge. A person who overcomes this barrier can be considered a very good Cmapper, once he/she is capable of modelling familiar content using a well-known graphical technique.

Excellent Cmappers (Level 3) are those who have already automated the process of modelling knowledge through concept mapping in their working memory. In this case, the E load should be as minimal as possible, and the resources are now available to deal with a high complex content (high I load). Subsequently, they should be able to produce high-quality concept maps, with an optimal graphical structure as well as content accuracy.

The last evolutionary stage of excellent Cmappers is to become professionals, who are proficient in the capture, organisation, representation and modelling of tacit knowledge from experts through the elicitation process (Hoffman, Shadbolt, Burton, \& Gary, 1995; Hoffman, Coffey, Ford, \& Novak, 2006). This level of expertise is only possible because the manipulation of the technique is totally automated for the Cmapper, being recovered to the working memory with a low cognitive resource investment. The available resources are entirely dedicated to dealing with the high I load imposed by the representation and modelling of content outside the individual's expertise. The effective use of concept maps in elicitation sessions has allowed the preservation and sharing of tacit and specialised knowledge (Moon, Hoffman, Novak, \& Cañas, 2011). It is important to highlight that the majority of users are not interested in becoming excellent or even professional Cmappers. In most cases, being a good Cmapper is sufficient for educational and even professional purposes.

\section{Prototyping sequential training oriented to certificate excellence in concept mapping}

Fig. 5 presents the prototype that organises the certification-oriented sequential training in concept mapping. The first training step is a computer-assisted and online course, which is organised in three sequential modules (proposition, hierarchy and focus question). Each module provides instruction interwoven with practice followed by an evaluation test. Instructional activities can be presented using digital resources, such as video lectures and audio descriptions. Practising allows content recall and hands-on activities to be presented as structured questionnaires, such as association, Cloze tests, multiple-choice questions and fill-in-the-blank. The users' performance in these exercises leads to accurate feedback during the learning process, even if the number of users is high.

In addition, the automation and customisation of feedback enables users to revisit instructions and perform extra exercises as required to fully understand the technique. Advancement to the next module is controlled by the performance on the assessment task (satisfactory performance), acting as learning set points. At the end of module 1 the user is invited to elaborate a concept map that is continuously revised at the end of modules 2 and 3, fostering metacognitive processes (Anderson \& Krathwohl, 2001). At the end of the course, the Cmappers should have the abilities for organising and representing 
knowledge through concept mapping (diagonal in Table 2). Satisfactory performance in the final course evaluation leads to user certification as a good Cmapper (Level 2).

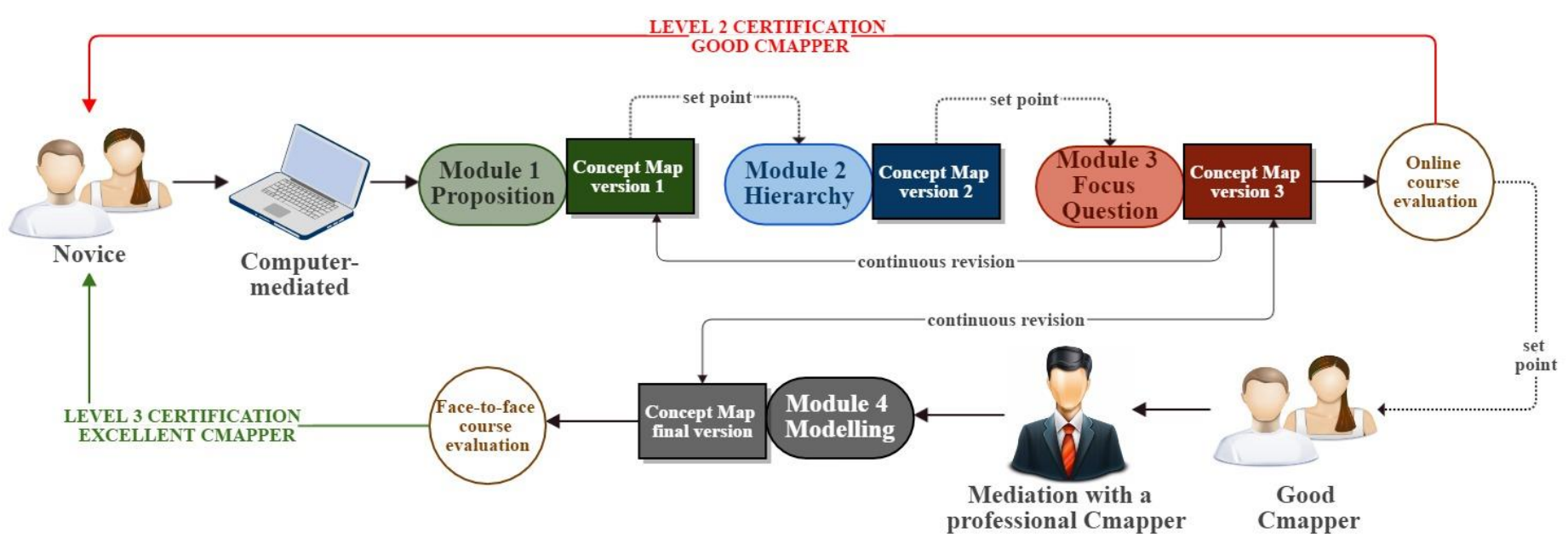

Fig. 5. Prototype that organises the certification-oriented sequential training in concept mapping

The second step is a face-to-face and expert-mediated course organised in a single module (modelling knowledge). Only good certified Cmappers can participate in this training stage, reducing the demand for this type of activity. In this module, instructions and practice are offered to teach how to refine and revise the concept map produced at the end of the online course. Collaboration, argumentation and self-explanation activities should ensure the development of modelling skills through concept mapping (Table 2). At the end of the course, the Cmappers prepare a concept map as a final evaluation test. Satisfactory performance, which means a concept map with excellent overall quality, structure and content, leads to user certification as an excellent Cmapper (Level 3).

\section{Final considerations}

We agree with Cañas, Novak, and Reiska (2015) that excellent concept maps are like good poems. However, professional Cmappers are challenged to (1) explain the differences between good and excellent concept maps (see Table 2) and (2) design thoughtful activities to teach novices how to master the concept mapping technique. The theoretical foundations from Bloom's revised taxonomy and cognitive load theory informed the two-step sequential training in concept mapping. The first step requires the fulfilment of low-order cognitive objectives (remember, understand, apply) to help novices to become good Cmappers through the development of knowledge representation skills. The second step requires the fulfilment of high-order cognitive objectives (analyse, evaluate, create) to help good Cmappers to become excellent ones through the development of knowledge modelling skills. The two-step training prototype allows us to massify the good use of concept mapping while providing a study plan for those interested in becoming excellent Cmappers. 
The current research on concept mapping is compatible with the proposal of certification of Cmappers, considering (1) the number of users of programs such as CmapTools (IHMC, Pensacola, USA) and (2) the maturity of the researchers that compose the international community of Cmappers. The application of this proposal and the institutional certification are the next steps for the mature use of this technique of knowledge management.

\section{Acknowledgements}

The authors thank FAPESP (São Paulo Research Foundation) for funding our research group (Grant\# 2012/22693-5). P.R.M.C. thanks FAPESP for funding his participation in the $7^{\text {th }}$ International Conference on Concept Mapping in Tallinn, Estonia (Grant\# 201609542-9). J.G.A. thanks CAPES for her PhD scholarship.

\section{References}

Aguiar, J. G., Cicuto, C. A. T., \& Correia, P. R. M. (2014). How can we prepare effective concept maps? Training procedures and assessment tools to evaluate mappers proficiency. Journal of Science Education, 15(1), 14-19.

Anderson, L. W., \& Krathwohl, D. R. (2001). A taxonomy for learning, teaching, and assessing: A revision of Bloom's taxonomy of educational objectives. New York: Longman.

Buhmann, S. Y., \& Kingsbury, M. (2015). A standardised, holistic framework for concept-map analysis combining topological attributes and global morphologies. Knowledge Management \& E-Learning, 7(1), 20-35.

Cañas, A. J., Leake, D. B., \& Wilson, D. C. (1999). Managing, mapping and manipulating conceptual knowledge. In Proceedings of the AAAI-99 Workshop on Exploring Synergies of Know Ledge Management and Case-Based Reasoning. Menlo Park: AAAI Press.

Cañas, A. J., \& Novak, J. D. (2006). Re-examining the foundations for effective use of concept maps. In Proceedings of the Second International Conference on Concept Mapping (Vol. 1, pp. 494-502). San Jose, Costa Rica: Universidad de Costa Rica.

Cañas, A. J., Novak, J. D., \& Reiska, P. (2015). How good is my concept map? Am I a good Cmapper? Knowledge Management \& E-Learning, 7(1), 6-19.

Cañas, A. J., Reiska, P., \& Novak, J. D. (2016). Is my concept map large enough? In A. J. Cañas, P, Reiska, \& J. D. Novak (Eds.), Innovating with Concept Mapping (pp. 128143). Springer.

Carvajal, R., Cañas, A. J., Carballeda, M., \& Hurtado, J. (2006). Assessing concept maps: First impressions count. In Proceeding of the Second International Conference on Concept Mapping (Vol. 1, pp. 28-31). San José, Costa Rica: Universidad de Costa Rica.

Coffey, J. W., Hoffmann, R. R., Cañas, A. J., \& Ford, K. M. (2002). A concept mapbased knowledge modeling approach to expert knowledge sharing. In Proceedings of the IKS 2002 - the IASTED international conference on information and knowledge sharing (pp. 212-217). Calgary, Canada: Acta Press.

Correia, P. R. M. (2012). The use of concept maps for knowledge management: From classrooms to research labs. Analytical and Bioanalytical Chemistry, 402(6), 19791986.

Correia, P. R M., \& Aguiar, J. G. (2014). Concept mapping informed by cognitive load theory: Implications for tasks involving learner-generated concept maps. In Proceedings of the Sixth International. Conference on Concept Mapping (pp. 150- 
157). Santos, Brazil.

De Aguiar, J. G., \& Correia, P. R. M. (2013). Como fazer bons mapas conceituais? Estabelecendo parâmetros de referência e propondo atividades de treinamento. Revista Brasileira de Pesquisa em Educação Ciências, 13(2), 141-157.

Derbentseva, N., \& Kwantes, P. (2014). Cmap readability: propositional parsimony, map layout and semantic clarity and flow. In Proceedings of the Sixth International. Conference on Concept Mapping. Santos, Brazil.

Fischer, F., \& Mandl, H. (2001). Facilitating the construction of shared knowledge with graphical representation tools in face-to-face and computer-mediated scenarios. In Proceedings of the euro-CSCL (pp. 230-236). McLuhan Institute, Maastricht.

Hilbert, T. S., \& Renkl, A. (2008). Concept mapping as a follow-up strategy to learning from texts: What characterizes good and poor mappers? Instructional Science, 36(1), 53-73.

Hoffman, R. R., Coffey, J. W., Ford, K. M., \& Novak, J. D. (2006). A method for eliciting, preserving, and sharing the knowledge of forecasters. Weather and Forecasting, 21(3), 416-428.

Hoffman, R. R., Shadbolt, N. R., Burton, A. M., \& Gary, K. (1995). Eliciting knowledge from experts: A methodological analysis. Organizational Behavior and Human Decision, 62(2), 129-158.

Hoffman, R. R., Ziebell, D., Fiore, S. M., \& Becerra-Fernandez, I. (2008). Knowledge management revisited. IEEE Intelligent Systems, 23(3), 84-88.

Kinchin, I. M., Hay, D. B., \& Adams, A. (2000). How a qualitative approach to concept map analysis can be used to aid learning by illustrating patterns of conceptual development. Educational Research, 42(1), 43-57.

Krathwohl, D. R. (2002). A revision of Bloom's taxonomy: An overview. Theory Into Practice, 41(4), 212-218.

Mayer, R. E. (2002). Rote versus meaningful learning. Theory Into Practice, 41(4), 226232.

Moon, B. M., Hoffman, R. R., Novak, J. D., \& Cañas, A. J. (2011). Applied concept mapping: Capturing, analyzing, and organizing knowledge. Boca Raton: CRC Press.

Newbern, D., \& Dansereau, D. F. (1995). Knowledge maps for knowledge management. In K. M. Wiig (Ed.), Knowledge Management Methods: Practical Approaches to Managing Knowledge. Arlington, TX: Schema Press.

Novak, J. D. (2010). Learning, creating, and using knowledge: Concept maps as facilitative tools in schools and corporations (2nd ed.). New York: Routledge.

Novak, J. D., \& Cañas, A. J. (2008). The theory underlying concept maps and how to construct and use them. Pensacola, FL: Institute for Human and Machine Cognition. Retrieved from http://cmap.ihmc.us/docs/theory-of-concept-maps

Safayeni, F., Derbentseva, N., \& Cañas, A. J. (2005). A theoretical note on concepts and the need for cyclic concept maps. Journal of Research in Science Teaching, 42(7), 741-766.

Sweller, J., Ayres, P., \& Kalyuga, S. (2011). Cognitive load theory. New York: Springer.

Tergan, S. O. (2005). Digital concept maps for managing knowledge and information. In S. O. Tergan \& T. Keller (Eds.), Knowledge and Information Visualization (pp. 185204). New York: Springer.

van Merriënboer, J. J. G., \& Ayres, P. (2005). Research on cognitive load theory and its design implications for e-learning. Educational Technology Research and Development, 53(3), 5-13.

van Mierlo, C. M., Jarodzka, H., Kirschner, F., \& Kirschner, P. A. (2012). Cognitive load theory in e-learning. In Z. Yan (Ed.), Encyclopedia of Cyber Behavior (pp. 11781211). EUA: Information Science Reference. 